\title{
Efeitos da adição de montmorilonita sódica na dieta sobre o perfil bioquímico de frangos de corte intoxicados com aflatoxina ${ }^{1}$
}

\author{
The effects of the addition of sodic montmorilonite on the feeding diet on the \\ biochemical profile of broiler chicken intoxicated by aflatoxin
}

\author{
Patricia Neves Batina ${ }^{2}$ Sonia Terezinha dos Anjos Lopes ${ }^{3}$ \\ Janio Moraes Santurio ${ }^{4}$ Cleverson de Souza ${ }^{5}$ Danieli Brolo Martins ${ }^{6}$
}

\section{RESUMO}

Este estudo teve como objetivo avaliar os valores bioquímicos e atividade enzimática hepática e renal em frangos de corte submetidos à intoxicação experimental com aflatoxina B1 com e sem a adição de montmorilonita sódica na dieta. Foram utilizados 528 frangos de corte, da linhagem Cobb, subtimetidos a seis tratamentos entre $1^{\circ}$ ao $42^{\circ}$ dia de vida: (T1) Controle: dieta normal; (T2) dieta com 5 ppm de aflatoxina; (T3) dieta com 0,25\% montmorilonita sódica; (T4) dieta com 5ppm de aflatoxina $+0,25 \%$ montmorilonita sódica; (T5) dieta com $0,5 \%$ montmorilonita sódica; (T6) dieta com 5ppm de aflatoxina $+0,5 \%$ montmorilonita sódica. O tratamento (T2) com aflatoxina demonstrou significante $(P<0,01)$ diminuição dos níveis de ácido úrico, albumina, colesterol, creatinina, triglicerídeos, globulinas e proteína total. Houve aumento significativo da alamino amino transferase (ALT) enquanto que a enzima aspartato amino transferase (AST) não foi significantemente diferente entre os tratamentos. Nos tratamentos T3 e T5, não houve alteração nas dosagens bioquímicas em relação ao tratamento controle (T1). A adição 0,5\% de montmorilonita sódica na dieta (T6) apresentou melhores resultados na redução da adsorção da aflatoxina em relação à adição de 0,25\% de montmorilonita sódica (T4). Os resultados obtidos, nas condições em que foi realizado este experimento, sugerem que altos níveis de aflatoxina (5ppm) na dieta causam alterações bioquímicas significativas na atividade enzimática de frangos de corte e que o tratamento preventivo com a montmorilonita sódica pode ser usado para se evitar o risco causado pela contaminação alimentar com aflatoxinas.

Palavras-chave: aflatoxina, montmorilonita sódica, frango de corte, perfil bioquímico.

\begin{abstract}
The aim of this research was to evaluate the hepatic biochemical profile and the renal enzymatic activity of broiler chicken experimentally intoxicated with aflatoxin and supplemented with sodic montmorilonite on the diet. 528 Cobb's broiler chickens were used divided in 6 treatments (from $1^{\text {st }}$ to the $42^{\text {th }}$ day of life) as described: (T1) control: normal diet; (T2) diet with 5ppm of aflatoxin; (T3) diet with $0.25 \%$ sodic montmorilonite; (T4) diet with 5 ppm of aflatoxin $+0.25 \%$ sodic montmorilonite; (T5) diet with $0.5 \%$ sodic montmorilonite; (T6) diet with 5 ppm of aflatoxin $+0.5 \%$ sodic montmorilonite. The treatment T2 showed significantly decreased levels of uric acid, albumin, cholesterol, creatinine, triglycerides, globulins and total serum proteins and an increase of serum alanine aminotransferase (ALT) activity. Aspartate aminotransferase (AST) did not showed important differences among treatments. In treatments $T 3$ and T5, there were no variations on the biochemical values, when compared to treatment T1. Addition of $0.5 \%$ of montmorilonite in the diet (T6), presented better results on the increase of the adsorption of aflatoxin when compared to $0.25 \%$ sodic montmorilonite (T4). Clearly, the results showed that high levels of aflatoxin in the diet (5ppm) causes important changes in the serum enzymatic activity of broiler chickens. Also, it was demonstrated that using sodic montmorilonite may be used to avoid risks caused by food contaminated by aflatoxins.
\end{abstract}

Key words: aflatoxin, sodic montmorilonite, broiler chicken, biochemistry profile.

${ }^{1}$ Dissertação de Mestrado do primeiro auto, apresentada ao Programa de Pós-graduação em Medicina Veterinária da Universidade Federal de Santa Maria (UFSM).

${ }^{2}$ Programa de Pós-graduação em Medicina Veterinária, Área de Clínica Médica de Pequenos Animais, Universidade Federal de Santa Maria (UFSM). Rua Pde Francisco Van Der Maas, 15-13, 17047-020, Bauru, SP, Brasil. E-mail: patricia@laborcare.com.br. ${ }^{3}$ Departamento de Clínica de Pequenos Animais, UFSM, Santa Maria, RS, Brasil.

${ }^{4}$ Departamento de Medicina Veterinária Preventiva, UFSM, Santa Maria, RS, Brasil.

${ }^{5}$ Aluno PhD da University of Minnesota, EUA.

${ }^{6}$ Aluno da Graduação do Curso de Medicina Veterinária da UFSM, Santa Maria, RS, Brasil. 


\section{INTRODUÇÃO}

As aflatoxinas estão amplamente presentes como contaminantes naturais dos alimentos. Quanto à composição química, as aflatoxinas são metabólicos tóxicos produzidos pelos fungos Aspergillus flavus, Aspergillus parasiticus e Aspergillus nominus (GIAMBRONE et al., 1985; BALACHANDRAN \& RAMAKRISHNAN, 1987). Os seres humanos e várias espécies domésticas são sensíveis aos seus efeitos tóxicos que podem ser agrupados como: agudos, mutagênicos, carcinogênicos e teratogênicos (EDDS \& BORDTELL, 1983).

O uso de alimentos contaminados pela aflatoxina, para fabricar rações, tem sido relatado como um problema importante e com sérias implicações econômicas para a indústria avícola, bem como de outras espécies domésticas de interesse econômico (BAILEY et al., 1998; KUBENA et al., 1998; LEDOUX et al., 1999; PARLAT et al., 1999) . A freqüência da contaminação dos produtos de consumo e a exposição crônica das aves a essas toxinas podem significar a diferença entre o lucro e prejuízo na indústria aviária (EDDS \& BORTELL, 1983; LEESON et al., 1995). A severidade das toxicoses depende da espécie e raças das aves, da idade, da quantidade e exposição à toxina, do estresse, e da composição nutricional da ração (LANZA et al., 1980).

Após ingeridas, as aflatoxinas são absorvidas no trato gastrintestinal e biotransformadas primariamente no fígado, por enzimas microssomais do sistema de oxidases mistas. O fígado é o órgão alvo da aflatoxicose nas aves. Os animais acometidos sofrem importantes alterações no metabolismo hepático afetando o metabolismo das proteínas, dos lipídeos, bem como a síntese e a cinética de algumas enzimas (TUNG et al., 1972).

Nos frangos, a aflatoxicose é caracterizada por anorexia, diminuição no crescimento, diminuição no ganho de peso e na produção de ovos, aumento na susceptibilidade a microrganismos comensais, estresse e aumento da mortalidade (BAILEY et al., 1998; KUBENA et al., 1998). Adicionalmente, pode se notar anemia (HUFF et al., 1988; KEÇECI et al., 1998), imunocomprometimento (CELIK et al., 1996; GABAL \& AZZAM, 1998), problemas hepáticos (EDRINGTON et al., 1997; KIRAN et al., 1998) e hemorragias (EDDS \& BORDTELL, 1983).

O conhecimento dos efeitos tóxicos da aflatoxina é importante para o diagnóstico das toxicoses dos frangos, sendo que as variações nos perfis bioquímico e hematológico podem ser utilizadas no diagnóstico desta condição (BRUGERE-PICOUX et al., 1987). A toxicidade da aflatoxina em frangos é caracterizada pela diminuição das concentrações de proteína total, albumina, colesterol, glicose, ácido úrico, fósforo inorgânico e cálcio e no aumento da atividade enzimática da alamino amino transferase (ALT) e da aspartato amino transferase (AST), indicativos de lesões hepáticas (AMER et al., 1998; SANTURIO et al., 1999). A enzima gama glutamiltransferase (GGT) tem baixa atividade no tecido hepático das aves. De acordo com HUFF et al., (1988), KUBENA et al. (1990b) e KEÇECI et al. (1998), a diminuição dos níveis séricos de proteína e albumina são indicadores patognomônicos de hepatotoxidade em frangos e perus devido à aflatoxicose.

Desde o início dos anos noventa, estudos têm sido dirigidos para o uso de adsorventes, naturais ou sintéticos, na tentativa de minimizar os efeitos da ingestão de alimento contaminado e da toxidade da aflatoxina nas aves (OGUZ et al., 2002). Segundo OLVER (1997), os adsorventes possuem a habilidade de aderir à aflatoxina e impedir sua absorção pelo trato gastrintestinal tornando-a inerte e não tóxica para os animais. Dentre os adsorventes que são utilizados comercialmente, estão os aluminosilicatos de $\mathrm{Na}$ e $\mathrm{Ca}$ (KUBENA et al., 1990b), as bentonitas (SANTURIO et al., 1999), os componentes da zeolitica (HARVEY et al., 1993) para o controle de aflatoxicose em rebanhos (KUBENA et al., 1990b; ABO-NORAG et al., 1995; KUBENA et al., 1998).

A montmorilonita sódica, pertence ao grupo das argilas, classificadas como Esmectitas, do grupo dos aluminosilicatos, é considerada promissora na absorção das aflatoxinas por reduzir os danos danos metabóbilicos em frangos de corte (ARABA \& WYATT, 1991; SCHEIDELER, 1993; SANTURIO et al., 1994; SANTURIO et al., 1999).

O objetivo deste estudo foi verificar possíveis alterações na atividade das enzimas séricas e substâncias bioquímicas que avaliam a função hepática e renal em aves experimentalmente intoxicadas pela aflatoxina e a eficiência do uso da montmorilonita sódica como medida de controle profilático nas intoxicações alimentares.

\section{MATERIAL E MÉTODOS}

Foram utilizadas 528 aves de linhagem Cobb, machos, provenientes do incubatório da Cooperativa Languiru de Teotônia (RS). As aves foram mantidas do $1^{\circ}$ até $42^{\circ}$ dia de vida, no Setor de Avicultura do Departamento de Zootecnia da Universidade Federal de Santa Maria. Os animais foram separados em 24 lotes, com 24 aves por box, 
divididos em 6 tratamentos. O programa de iluminação utilizado foi de 24 horas de luminosidade, nos primeiros 21 dias de idadeApós foi utilizada apenas iluminação natural com o objetivo de reduzir o estresse das aves. A ração foi formulada de acordo com a fase de idade das aves, conforme a tabela 1. A água foi fornecida ad libitum durante todo o experimento.

O experimento foi constituído de seis tratamentos: (T1) Controle: dieta normal, (T2): dieta com $5 \mathrm{ppm}$ de aflatoxina, (T3): dieta com $0,25 \%$ de montmorilonita sódica, (T4): dieta com 5ppm de aflatoxina $+0,25 \%$ de montmorilonita sódica, (T5): dieta com 0,5\% montmorilonita sódica e (T6): dieta com 5ppm de aflatoxina $+0,5 \%$ montmorilonita sódica.

A aflatoxina foi produzida pelo Laboratório de Pesquisas Micológicas (LAPEMI) - UFSM a partir da fermentação controlada do fungo Aspergillus parasiticus, cepa NRRL 2999 em arroz e a montmorilonita sódica (Scamix Plus ${ }^{\circledR}$ ) foi fornecido pela empresa Scavet ${ }^{\mathrm{a}}$.

Quando os frangos atingiram 42 dias de idade, foram selecionados 4 aves de cada lote, ou seja, 96 aves por tratamento, e coletados $10 \mathrm{ml}$ de sangue, sem anticoagulante, por punção cardíaca após desensibilização por corrente elétrica. O soro obtido após a centrifugação do sangue foi estocado a $-20^{\circ} \mathrm{C}$ para posterior análise das concentrações de ácido úrico, albumina, proteínas totais, aspartato aminotransferase (AST), alanina aminotransferase (ALT), colesterol, creatinina, triglicerídeos e uréia. As determinações bioquímicas foram realizadas por meio de analisador semi-automático Bio-200F, Bioplus $\AA^{\mathrm{b}}$ com uso de kits comerciais ${ }^{\mathrm{c}}$. Os dados obtidos foram

Tabela 1 - Composição estimada das dietas basais da fase inicial, crescimento e final de frangos de corte.

\begin{tabular}{lccc}
\hline & \multicolumn{3}{c}{ Fases } \\
\cline { 2 - 4 } Composição química & Inicial & Crescimento & Final \\
& $(1-21$ dias $)$ & $(22-36$ dias $)$ & $(36-42$ dias $)$ \\
\hline Proteína bruta (\%) & 22,00 & 21,00 & 21,00 \\
Energia metabolizave $)$ & 3050 & 3100 & 3100 \\
(Kcal kg $\left.{ }^{-1}\right)$ & 1,00 & 0,96 & 0,96 \\
Cálcio (\%) & 0,45 & 0,45 & 0,45 \\
Fósforo útil (\%) & 0,22 & 0,22 & 0,22 \\
Sódio (\%) & 1,30 & 1,26 & 1,26 \\
Lisina (\%) & 0,56 & 0,54 & 0,54 \\
Metionina (\%) & 0,92 & 0,89 & 0,89 \\
Metionina + Cisteina & $\%)$ & 0,80 & 0,80 \\
Treonina (\%) & 0,80 & 0,20 & 0,20 \\
Triptofano (\%) & 0,20 & & \\
\hline
\end{tabular}

analisados estatisticamente usando-se o teste estatístico One Way - Anova - Tukey-Kramer Test. Os dados que não apresentaram distribuição normal segundo o teste de Kolmogorov Smirnov foram transformados para a base de Log10. Os valores classificados como "outliers" foram retirados do experimento, visto que os mesmos poderiam comprometer a análise das médias. O pacote estatístico utilizado foi o NCSS $® 2001^{\mathrm{d}}$. Os resultados com valores de $\mathrm{P}<0,01$ foram considerados estatisticamente significativos.

\section{RESULTADOS E DISCUSSÃO}

Os resultados obtidos estão mostrados na tabela 2. Estes dados mostram o efeito da montmorilonita sódica em aves intoxicadas com aflatoxina e alterações nos valores bioquímicos séricos em frangos com 42 dias de idade.

A dieta somente com aflatoxina tratamento T2 causou diminuição dos níveis séricos de ácido úrico, colesterol, creatinina e triglicérideos $(\mathrm{P}<0,01)$. Ocorreu aumento dos níveis de ALT, indicando lesão hepática, o que está de acordo com AMER et al. (1998) e SANTURIO et al. (1999) que encontraram aumento dessa enzima em frangos intoxicados com aflatoxina. Segundo KUBENA et al. (1998), o aumento da atividade da ALT e AST são considerados indicadores de alta sensibilidade da disfunção/lesão e inflamação hepática e lesão ou obstrução dos ductos biliares.

Para a AST, não houve alterações significativas nos níveis séricos, neste trabalho, contrariando os resultados obtidos por AMER et al. (1998) e SANTURIO et al. (1999) em experimentos semelhantes.

No experimento tratamento T2, houve diminuição nos níveis de proteínas séricas totais e albumina. TUNG et al. (1972) também observaram a diminuição dos níveis de proteína total e albumina semelhante ao encontrado no presente experimento. Esta diminuição é atribuída ao bloqueio da síntese de RNA e inibição da síntese protéica no fígado causada pela aflatoxina, sendo estes dados sensíveis indicadores das aflatoxicoses (CLIFFORD \& REES, 1967). Quanto às imunoglobulinas, houve diminuição nos níveis séricos no tratamento T2. Embora não sendo significativa, demonstra possível imunocomprometimento conforme citam CELIK et al. (1996) e GABAL \& AZZAM (1998). No entanto, os tratamentos T4 e T6 apresentaram aumento nos comparados ao tratamento T1. Estes dados demonstram que a montmorilonita sódica foi capaz de estimular o sistema imune em frangos acometidos por aflatoxicose. 
Tabela 2 - Efeitos da aflatoxina e montmorilonita sódica (MMS) na dieta de frangos de corte sobre os níveis séricos de ácido úrico, albumina, ALT, AST, colesterol, creatinina, triglicérides, globulinas, proteína total e uréia.

\begin{tabular}{|c|c|c|c|c|c|c|c|c|c|c|c|}
\hline \multicolumn{2}{|c|}{ DIETA } & \multicolumn{10}{|c|}{ METABÓLICOS } \\
\hline $\begin{array}{l}\text { Aflatoxina } \\
\text { (ppm) }\end{array}$ & $\begin{array}{c}\text { a MMS } \\
(\%)\end{array}$ & $\begin{array}{l}\text { Ácido úrico } \\
\left(\mathrm{mg} \mathrm{dL}^{-1}\right)\end{array}$ & $\begin{array}{c}\text { Albumina } \\
\left(\mathrm{g} \mathrm{dL}^{-1}\right)\end{array}$ & $\begin{array}{c}\mathrm{ALT} \\
\left(\mathrm{U} \mathrm{L}^{-1}\right)\end{array}$ & $\begin{array}{c}\mathrm{AST} \\
\left(\mathrm{UL}^{-1}\right)\end{array}$ & $\begin{array}{l}\text { Colesterol } \\
\left(\mathrm{mg} \mathrm{dL}^{-1}\right)\end{array}$ & $\begin{array}{l}\text { Creatinina } \\
\left(\mathrm{mg} \mathrm{dL}^{-1}\right)\end{array}$ & $\begin{array}{l}\text { Globulinas } \\
\left(\mathrm{g} \mathrm{dL}^{-1}\right)\end{array}$ & $\begin{array}{l}\text { Proteína Total } \\
\qquad\left(\mathrm{g} \mathrm{dL}^{-1}\right)\end{array}$ & $\begin{array}{l}\text { Triglicerídeos } \\
\left(\mathrm{mg} \mathrm{dL}^{-1}\right)\end{array}$ & $\begin{array}{c}\text { Uréia } \\
\left(\mathrm{mg} \mathrm{dL}^{-1}\right)\end{array}$ \\
\hline 0 & 0 & $5,6^{\mathrm{a}, \mathrm{c}}$ & $1,26^{\mathrm{a}}$ & $5,05^{\mathrm{a}}$ & $234,94^{\mathrm{a}}$ & $142,24^{\mathrm{a}, \mathrm{c}}$ & $0,48^{\mathrm{a}}$ & $2,45^{\mathrm{a}}$ & $3,71^{\mathrm{a}}$ & $88,95^{\mathrm{a}, \mathrm{b}, \mathrm{c}}$ & $6,55^{\mathrm{a}, \mathrm{b}}$ \\
\hline 5 & 0 & $5,5^{\mathrm{a} . \mathrm{b}}$ & $0,61^{\mathrm{b}}$ & $9,71^{\mathrm{b}, \mathrm{c}}$ & $210,28^{\mathrm{a}}$ & $93,82^{b, c}$ & $0,34^{\mathrm{b}}$ & $1,84^{\mathrm{a}}$ & $2,46^{\mathrm{b}}$ & $64,98^{\mathrm{b}}$ & $5,46^{\mathrm{b}}$ \\
\hline 0 & 0.25 & $5,9^{\mathrm{a}}$ & $1,18^{\mathrm{a}}$ & $4,81^{\mathrm{a}}$ & $221,85^{\mathrm{a}}$ & $134,5^{\mathrm{acc}}$ & $0,54^{\mathrm{a}}$ & $2,53^{\mathrm{a}}$ & $3,69^{\mathrm{a}}$ & $91,31^{\mathrm{a}, \mathrm{c}}$ & $7,28^{\mathrm{a}}$ \\
\hline 5 & 0.25 & $4,3^{\mathrm{b}}$ & $0,71^{\mathrm{b}}$ & $10,3^{\mathrm{c}}$ & $235,5^{\mathrm{a}}$ & $88,7^{\mathrm{b}}$ & $0,46^{\mathrm{a}, \mathrm{b}}$ & $3,92^{\mathrm{b}}$ & $4,64^{\mathrm{a}}$ & $72,39^{\mathrm{a}, \mathrm{b}}$ & $8,4^{c}$ \\
\hline 0 & 0.5 & $6,0^{\mathrm{a}}$ & $1,16^{\mathrm{a}}$ & $4,59^{\mathrm{a}}$ & $229,7^{\mathrm{a}}$ & $151,2^{\mathrm{a}}$ & $0,59^{\mathrm{a}}$ & $2,49^{\mathrm{a}}$ & $3,66^{\mathrm{a}}$ & $100,56^{\mathrm{c}}$ & $7,76^{\mathrm{a}, \mathrm{c}}$ \\
\hline 5 & 0.5 & $7,5^{\mathrm{c}}$ & $1,0^{\mathrm{a}}$ & $6,81^{a, b}$ & $226,17^{\mathrm{a}}$ & $117,04^{\mathrm{c}}$ & $0,5^{\mathrm{a}}$ & $5,55^{\mathrm{c}}$ & $6,55^{\mathrm{c}}$ & $88,6^{\mathrm{a}, \mathrm{b}, \mathrm{c}}$ & $7,47^{\mathrm{a}, \mathrm{c}}$ \\
\hline
\end{tabular}

${ }^{a}$ Para letras iguais não há diferença significativa $(\mathrm{P}<0,01)$ entre os momentos, de acordo com o teste One Way - Anova Tukey-Kramer Test.

Nos tratamentos T3 e T5 que apresentavam adição de montmorilonita sódica $(0,25 \%$ e $0,5 \%$, respectivamente), observam-se resultados similares ao tratamento $\mathrm{T} 1$, o que era de se esperar, haja vista que o adjuvante utilizado não tem efeito sobre a dieta ou biodisponibilização de nutrientes.

A adição de $0,25 \%$ de montmorilonita sódica à dieta com aflatoxina (T4) não promoveu alteração dos níveis bioquímicos em relação às aves intoxicadas com aflatoxina tratamento (T2), para a maioria dos níveis bioquímicos analisados, com exceção das globulinas, proteínas séricas totais e uréia que foram significativamente mais elevados em tratamento T4. A diminuição dos níveis séricos de ácido úrico e colesterol nos tratamentos T2 e T3, possivelmente, está relacionada ao metabolismo hepático e ao transporte de lipídeos intrahepático que são prejudicados pela aflatoxina. Estes resultados vêm de acordo com os dados descritos por KUBENA et al. (1998); KEÇECI et al. (1998); OGUZ et al. (2000a) e REDDY et al. (1984), que encontraram resultados similares em pesquisa com frangos de corte.

Já a adição de montmorilonita sódica $(0,5 \%)$ na dieta com aflatoxina (5ppm) em tratamento T6 impediu a diminuição dos níveis séricos da albumina, uréia, creatinina, colesterol, triglicerídeos e proteínas séricas, que se aproximaram aos níveis apresentados pelo controle (tratamento T1). Os resultados deste grupo demonstram que a utilização do adsorvente inibiu a absorção da aflatoxina no sistema gastrointestinal reduzindo os efeitos da aflatoxicose nos frangos de corte, o que está de acordo com os resultados de SANTURIO et al. (1999) e OGUZ et al. (2000) que mostraram a importância da utilização dos adsorventes na dieta de frangos de corte, diminuindo o impacto econômico causado pela aflatoxina. No tratamento T6, observa-se que houve melhora nos níveis séricos da albumina, ALT, creatinina, triglicérideos e proteína total em relação ao tratamento T2. Estes resultados foram similares a outros adsorventes largamente utilizados na indústria aviária como as bentonitas (SANTURIO et al. 1999) e aluminosilicatos (KUBENA et al. 1990b).

\section{CONCLUSÃO}

Os resultados obtidos, nas condições em que foi realizado este experimento, mostraram que a presença de aflatoxina na dieta de frangos de corte causa várias alterações bioquímicas, notadamente, com relação ao tecido hepático.

A concentração de $0,25 \%$ montmorilonita sódica não reduziu o efeito da absorção da aflatoxina, constatado pelas alterações dos níveis bioquímicos séricos iguais à dieta com aflatoxina (5ppm). Já a concentração de $0,5 \%$ montmorilonita sódica na dieta determinou um efeito benéfico, prevenindo a absorção da aflatoxina, demonstrado pelos níveis bioquímicos séricos semelhantes ao controle, provando assim sua eficácia na prevenção dos efeitos da aflatoxicose em frangos de corte.

\section{AGRADECIMENTOS}

Setor de Avicultura do Departamento de Zootecnia da UFSM-RS, Laboratório de Patologia Clínica do Hospital Veterinária de Santa Maria-RS

\section{FONTESDEAQUISIÇÃO}

a. Sacvet - Comércio e Representações de Importação e Exportação de Produtos Vet. Ltda.

b. Bio-200F, Bioplus ${ }^{\circledR}$

c. Celm ${ }^{\circledR}$, São Paulo, Brasil.

d. Resultados do NCSS ${ }^{\circledR} 2001$ Tukey-Kramer MultipleComparison Test.

Ciência Rural, v.35, n.4, jul-ago, 2005. 


\section{REFERÊNCIAS}

ABO-NORAG, M. et al. Influence of hydrated sodium calcium aluminosilicate and virginiamycin on aflatoxicosis in broiler chicks. Poultry Science, v.74, p.626-632, 1995.

AMER, A.M.M. et al. Effect of aflatoxicosis on kinetic behaviour of ceftiofur in chickens. Research Veterinary Science, v.65, p.115-118, 1998.

ARABA, M.; WYATT, R.D. Effects of sodium bentonite, hydrated sodium calcium alumino-silicate $\left(\right.$ Novasil $\left.^{\mathrm{TM}}\right)$, and ethical on aflatoxicosis in broiler chickens. Poultry Science, v.70, p.6, 1991.

BAILEY, R.H. et al. Efficacy of various inorganic sorbents to reduce the toxicity of aflatoxin and T-2 toxin en broiler chickens. Poultry Science, v.77, p.16231630,1998 .

BALACHANDRAN, C.; RAMARKRISHNAN, R. Influence of dietary aflatoxin on certain serum enzyme levels in broiler chickens. Mycopathologia, v.101, p.65-67, 1987.

BRUGERE-PICOUX, J. et al. Biochimie clinique en pthologie aviaire. Interet et limites des dosages enzymatiques ches $b$ poule. Recuel Medicine Vetereneri, v.163, p.1091-1099, 1987.

CELIK, I. et al. Determination of phagocytic and candidacidal activities of peritoneal macrophages isolated from chickens fed with aflatoxin and aflatoxin adsorbing agent, polyvinylpolypyrroline. Journal of Veterinary Science, v.12, p.145-151, 1996.

CLIFFORD, J.I.; RESS, K.R. Aflatoxin: a site of action in the rat liver cell. Nature, v.209, p.312-313, 1967.

EDDS, G.T.; BORTELL, R.A. Biologycal effects of aflatoxins: poultry toxin and Aspergillus flavus in corn. In: DIERNER, U.L. et al. Bulletin of Alabama Agricola Experiment Station, Alabama AL : Auburn University, 1983. p.64-66.

EDRINGTON, T.S. et al. Influence of superativated charcoal on the toxic effects of aflatoxin and $\mathrm{T} 2$ toxin in growing broilers. Poultry Science, v.76, p.1205-1211, 1997.

GABAL, M.A.; AZZAM, A.H. Interaction of aflatoxin en the feed and immunization against selected infectious diseases in poultry. II Effect on one-day-old layer chicks simultaneously vaccinated against Newcastle disease infectious bronchitis and infectionus bursal disease. Avian Pathology, v.27, p.290-295, 1998.

GIAMBRONE, J.J. et al. Effects of purified aflatoxin on broiler chickens. Poultry Science, v.64, p.852-858, 1985 .

HARVEY, R.B. et al. Efficacy of zeolitic ore compounds on the toxicity of aflatoxin to growing broiler chickens. Avian Diseases, v.37, p.67-73, 1993.

HUFF, W.E. et al. Toxic synergism between aflatoxin and T2 toxin in broiler chicken. Poultry Science, v.67, p.1418$1420,1988$.
KEÇECI, T. et al. Effects of polyvinylpolyrroline, synthetic zeolite and betonite on serum biochemical and haematological charactes of broiler chickens during aflatoxicosis. British Poultry Science, v.39, p.452-458, 1998.

KIRAN, M.M. et al. The preventive effect of polyvinylpolypyrrolidone on aflatoxicosis in broilers. Avian Pathology, v.27, p.250-255, 1998.

KUBENA, L.F. et al. Diminution of aflatoxicosis in growing chickens by the dietary addition of hydrated, sodium aluminosilicate. Poultry Science, 69, p.727-735, 1990b.

KUBENA, L.F. et al. Effects of hydrated sodium calcium aluminosilicate (T-Bind ${ }^{\mathrm{TM}}$ ) on mycotoxicosis in young broiler chickens. Poultry Science, v.77, p.1502-1509, 1998 .

LANZA, G.M. et al. Strain variation in hematological response of broilers to dietary aflatoxin. Poultry Science, v.59, p.2686-2691, 1980a.

LEDOUX, D.R. et al. Efficacy of hydrated sodium calcium aluminosilicate to ameliorate the toxic effects of aflatoxin in broiler chicks. Poultry Science, v.78, p.204-210, 1999.

LEESON, S. et al. Aflatoxins. In: LEESON, S. Mycotoxins. Ontario, Canada : University Books, 1995. p.248-279.

OGUZ, H. et al. Effect of clinoptiolite on serum biochemical and haematological characters of broiler chickens during experimental aflatoxicosis. Research Veterinary Science, v.69, p. 89-93, 2000a.

OGUZ H. et al. Preventive efficacy of clinoptilolite in broilers during chronic aflatoxin (50 and $100 \mathrm{ppb}$ ) exposure. Research Veterinary Science, v.69, p.197$201,2000$.

OGUZ H, et al. Evaluation of biochemical characters of broiler chickens during dietary aflatoxin (50 and $100 \mathrm{ppb}$ ) and clinoptilolite exposure. Research Veterinary Science, v.73, p.101-103, 2002.

OLVER, M.D. Effect of feeding clinoptilolite (zeolite) on the performance of three strains of laying hens. British, Poultry Science, v.38, p.220-222, 1997.

PARLAT, S.S. et al. Effects of clinoptilolite on fattening performece of Japanese quail (Coturinix coturnix japonica) during experimental aflatoxicosis. Britisch Poultry Science, v.40, p.495-500, 1999.

REDDY, D.N. et al. Effect of selected levels of dietary aflatoxin on the performance of broiler chicken. Indian Journal Animal Science, v.54, p.68-73, 1984.

SANTURIO, J.M. et al. Níveis de adsorção de aflatoxina B 1 in vitro de aluminosilicatos e betonitas comercializadas no Brasil. In: CONGRESSO LATINO AMERICANO DE MICOTOXICOLOGIA, 1994, Rio de Janeiro. Proceedings... Rio de Janeiro, 1994. p.10-11. 
SANTURIO, J.M. et al. Effect of sodium bentonite on the performance and blood variables of broiler chickens intoxicated with aflatoxins. British Poultry Science, v.40, p.115-119, 1999.

SCHEIDELER, S.E. Effects of various types of aluminosilicates and aflatoxin B1 on aflatoxin toxicity, chick performance, and mineral status. Poultry Science, v.72, p.282-288, 1993

TUNG, H.T. et al. Altered lipid transport during aflatoxicosis. Toxicology and Applied Pharmcology, v.22, p.795-800, 1972 . 